\title{
Ventricular fibrogenesis activity assessed by serum levels of procollagen type III N-terminal amino peptide during the staged Fontan procedure
}

\author{
Masaya Sugimoto, MD, ${ }^{\mathrm{a}, \mathrm{b}}$ Hirofumi Saiki, MD, ${ }^{\mathrm{a}}$ Akiko Tamai, MD, ${ }^{\mathrm{a}}$ Mitsuru Seki, MD, \\ Ryo Inuzuka, MD, ${ }^{\mathrm{c}}$ Satoshi Masutani, MD, ${ }^{\mathrm{a}}$ and Hideaki Senzaki, $\mathrm{MD}^{\mathrm{a}}$
}

\begin{abstract}
Objective: We tested the hypotheses that volume overload and cyanosis observed in the pre-Fontan single ventricular circulation are associated with increased ventricular fibrogenesis, that the Fontan procedure helps to reduce fibrogenesis, and that persistently increased fibrogenesis in the Fontan ventricle is associated with ventricular diastolic dysfunction.
\end{abstract}

Methods: Levels of serum amino-terminal procollagen type III, a marker of tissue fibrogenesis, were measured in 172 patients with single ventricle circulation and 149 controls. Patients were divided into 3 groups according to surgical stage: 59 patients after Blalock-Taussig shunt or pulmonary banding, 60 patients after Glenn surgery (Glenn group), and 53 patients after Fontan surgery (Fontan group).

Results: Serum amino-terminal procollagen type III levels were significantly higher among the 3 single ventricle groups than among control patients, but decreased with each surgical stage $(0.604,0.176,0.143$, and $0.073 \mathrm{U} / \mathrm{mL}$, for Blalock-Taussig shunt or pulmonary banding, Glenn, Fontan, and controls, respectively). Severity of volume load and cyanosis were independent determinants of increased amino-terminal procollagen type III levels in patients before Fontan surgery, and persistently increased amino-terminal procollagen type III after Fontan surgery was associated with ventricular diastolic stiffening $(r=0.494, P=.009)$. Data also indicated close associations between amino-terminal procollagen type III levels and activation of the reninangiotensin-aldosterone system, suggesting potential involvement of this hormonal system in the increased fibrogenesis after Fontan surgery.

Conclusions: These results suggest that serum amino-terminal procollagen type III may provide important diagnostic information on myocardial fibrosis in patients with single ventricle circulation and raise the possibility that ventricular fibrogenesis may be a potential therapeutic target in this population. (J Thorac Cardiovasc Surg 2016;151:1518-26)

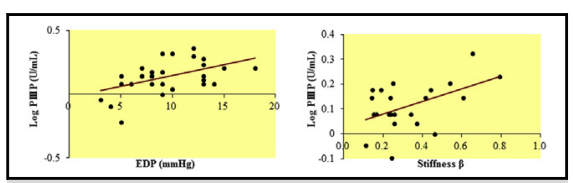

PIIIP levels are associated with ventricular diastolic dysfunction.

\section{Central Message}

Serum procollagen type III may provide useful diagnostic and therapeutic information to improve prognosis in patients undergoing the Fontan procedure.

\section{Perspective}

Increased ventricular volume load and cyanosis before the Fontan procedure are associated with increased ventricular fibrotic activity. The Fontan procedure ameliorates this process, but ongoing fibrogenesis activation in some patients undergoing the Fontan is related to ventricular diastolic dysfunction. The effects of therapies that potentially suppress ventricular fibrogenesis warrant future study.

See Editorial Commentary page 1527.
From the ${ }^{a}$ Department of Pediatric Cardiology, Saitama Medical Centre, Saitama Medical University, Kawagoe, ${ }^{\mathrm{b}}$ Department of Pediatrics, Asahikawa Medical University, Asahikawa, and ${ }^{\mathrm{c}}$ Department of Pediatrics, University of Tokyo, Tokyo, Japan.

Received for publication July 10, 2015; revisions received Dec 23, 2015; accepted for publication Jan 11, 2016; available ahead of print Feb 28, 2016.

Address for reprints: Hideaki Senzaki, MD, Department of Pediatric Cardiology Saitama Medical Center, Saitama Medical University, Staff Office Building 415, 1981 Kamoda, Kawagoe, Saitama, 350-8550 Japan (E-mail: hsenzaki@ saitama-med.ac.jp).

$0022-5223 / \$ 36.00$

Copyright $₫ 2016$ Published by Elsevier Inc. on behalf of The American Association for Thoracic Surgery

http://dx.doi.org/10.1016/j.jtcvs.2016.01.020
Although the Fontan procedure has greatly contributed to the improvement of mortality rates in patients with a single ventricle (SV) circulation, adverse outcomes become increasingly prevalent with time after this procedure. ${ }^{1-3}$

Scanning this QR code will take you to the article title page. 


$$
\begin{aligned}
& \text { Abbreviations and Acronyms } \\
& \begin{aligned}
\text { ANOVA } & =\text { analysis of variance } \\
\mathrm{BNP} & =\text { brain natriuretic peptide } \\
\mathrm{BTS} & =\text { Blalock-Taussig shunt } \\
\mathrm{EDP} & =\text { end-diastolic pressure } \\
\mathrm{PAB} & =\text { pulmonary artery banding } \\
\mathrm{PIIP} & =\text { amino-terminal procollagen type III } \\
\mathrm{Qp} / \mathrm{Q} & =\text { pulmonary-to-systemic blood flow } \\
\mathrm{RAS} & =\text { renin-angiotensin-aldosterone system } \\
\mathrm{SaO} & =\text { arterial oxygen saturation } \\
\mathrm{SV} & =\text { single ventricle }
\end{aligned}
\end{aligned}
$$

Ventricular dysfunction is one such adverse outcome, but the precise mechanisms for progression of ventricular dysfunction after Fontan surgery remain poorly understood. Collagen deposition in the myocardium (ventricular fibrosis) is a key feature of ventricular remodeling associated with ventricular dysfunction and failure, ${ }^{4,5}$ and abnormal hemodynamic load and hypoxia (cyanosis) are known to be important stimuli that promote cardiac fibrosis. ${ }^{6,7}$ During the staged interventions toward the Fontan procedure, various degrees of ventricular volume load and cyanosis coexist and markedly change with each palliative surgical stage. Therefore, it is possible that ventricular fibrogenic activity is enhanced in patients with SV circulation and is altered with advancing the palliative stage. In addition, persistently increased ventricular fibrogenesis even after amelioration of volume load and cyanosis with the Fontan procedure may be related to ventricular dysfunction.

To test these hypotheses, we assessed ventricular fibrogenic activity by measuring serum levels of amino-terminal procollagen type III (PIIIP), a useful marker of tissue fibrogenesis that correlates with myocardial collagen type III contents. ${ }^{8-10}$ Specifically, we tested whether volume overload or cyanosis observed in the pre-Fontan SV circulation is associated with increased ventricular fibrogenesis in patients before the Fontan procedure, whether enhanced fibrogenesis is effectively reduced by the Fontan procedure, which ameliorates fibrogenic stimuli (volume overload and cyanosis), and whether a persistent increase in fibrogenesis in the Fontan ventricle is associated with ventricular diastolic dysfunction.

\section{MATERIALS AND METHODS \\ Patients}

We enrolled 172 consecutive patients aged 10 years or less with an SV circulation who underwent cardiac catheterization and 149 volunteer children with noncardiac conditions (controls) aged 10 years or less who had been admitted to affiliated local hospitals. We selected this age group to avoid the effects of the age-associated nonlinear fluctuation of PIIIP levels on the analysis, because a previous report ${ }^{11}$ and our own data indicated that PIIIP levels decrease linearly with age after birth but increase significantly later at puberty (aged $\sim 10$ years), and then decrease thereafter, reaching levels similar to those in adults by 16 years of age. Patients with SV circulation were divided into 3 groups according to their clinical stages as follows: 59 had received a first-stage repair (Blalock-Taussig shunt [BTS] or pulmonary artery banding [PAB]; BTS/PAB group), 60 had undergone a bidirectional Glenn anastomosis or hemi-Fontan surgery as a second-stage palliation (Glenn group), and 53 had undergone the Fontan procedure (Fontan group). Before the Fontan surgery, all patients underwent cardiac catheterization for preoperative evaluation of the next surgical stage (ie, patients with BTS/ PAB for the Glenn and patients with the Glenn for the Fontan surgery). Catheterization was also performed in patients undergoing the Fontan for the postoperative evaluation of Fontan hemodynamics, as is standard clinical practice in Japan and in our institutions. All study subjects had undergone appropriate clinical and laboratory evaluations to exclude other conditions associated with elevated serum PIIIP levels (eg, chronic liver disease, renal failure, pulmonary fibrosis, and extensive wounds). Control group children had been admitted to local hospitals affiliated to Asahikawa Medical University for the treatment of respiratory tract infections, asthma, or epilepsy, but had fully recovered from their illness at the time of study enrolment. Written informed consent was obtained from the parents of all patients, and the procedures were approved by the Committee on Clinical Investigation of the respective institutions (Saitama Medical University No. 13-034 and No. 972, and Asahikawa Medical University No. 1706).

\section{Hemodynamic Measurements}

Pressure measurement and blood sampling for the measurement of oxygen saturation were performed during catheterization, followed by angiography/ventriculography as necessary. The ratio of the pulmonary-to-systemic blood flow ( $\mathrm{Qp} / \mathrm{Qs})$ was derived from oximetric measurements by applying Fick's principle. Ventricular pressure-area loops and relationships during caval occlusion were constructed in 22 randomly selected patients in the Fontan group to examine ventricular diastolic passive stiffness. The details of this methodology have been described. $^{2}$

\section{Measurement of the Serum Amino-Terminal Procollagen Type III Levels and the Renin- Angiotensin-Aldosterone System}

The serum PIIIP levels were measured from blood samples obtained from the inferior vena cava during cardiac catheterization. Blood samples were immediately centrifuged after sampling, and the serum was stored at $-80^{\circ} \mathrm{C}$ for subsequent analysis. The serum PIIIP levels were measured by immunoradiometry using a mouse monoclonal antibody and an IRMA kit for PIIIP measurement (Riagnost P-III-P, CIS Bio International, Ceze, France). ${ }^{12}$ The inter- and intra-assay variations were $7 \%$ and $3 \%$, respectively. To evaluate the status of the renin-angiotensin-aldosterone system (RAS), a potentially important hormonal system for inducing cardiac fibrosis, ${ }^{13}$ plasma levels of renin, angiotensin II, and serum aldosterone levels were measured using commercially available radioimmunoassay kits (Renin; FUJIREBIO Inc, Tokyo, Japan; Angiotensin2 set; FUJIREBIO Inc, SPAC-S Aldosterone Kit; FUJIREBIO Inc, respectively).

\section{Statistical Analysis}

All data were expressed as mean \pm standard deviation. Spearman's rank correlation coefficient was used to assess the intervariable relationships. Serum PIIIP levels, expressed as log-transformed values, were compared between patients in the SV groups and control patients by analysis of variance (ANOVA) followed by the Dunnett's post hoc multiple comparison test. Stepwise multivariate linear regression analysis was performed to test the effects of age, hemodynamic load, and cyanosis on the serum PIIIP 
levels. Correlation between continuously distributed variables was examined by linear regression analysis. A nonlinear effect of $\mathrm{Qp} / \mathrm{Qs}$ on PIIIP levels in the BTS/PAB group was tested by the random forests method. ${ }^{14}$ All analyses were performed using a commercially available statistical software package (SPSS for Windows, Version 22.0, IBM, New York, NY), except for the random forests analysis, which was carried out with R Version 3.0.1 and the package "random Forest SRC."

\section{RESULTS}

Table 1 summarizes the characteristics of patients in each group, including demographic data, underlying cardiac lesions, and hemodynamics. The mean age of patients in the Fontan group was greater than that of those in the $\mathrm{BTS} / \mathrm{PAB}$ and Glenn groups because of the nature of staged palliations, but was comparable to that of the controls. Distribution of the underlying cardiac lesion was similar among the 3 groups with SV circulation. The BTS/PAB group showed increased pulmonary flow, as evidenced by a mean $\mathrm{Qp} / \mathrm{Qs}$ ratio of 1.6 , demonstrating a ventricular volume overload. The arterial oxygen saturation $\left(\mathrm{SaO}_{2}\right)$ levels in the BTS/PAB and Glenn groups were significantly lower than those in the Fontan group $(P<.05)$. The mean brain natriuretic peptide (BNP) levels in the BTS/PAB, Glenn, and Fontan groups were significantly higher than those in the controls $(P<.05)$, reflecting the ventricular loading condition of each stage of SV circulation.

\section{Serum Amino-Terminal Procollagen Type III Levels}

Figure 1 shows age-associated changes in serum PIIIP levels in our control patients, demonstrating that PIIIP levels significantly decreased with advancing age (log
PIIIP $=-0.024 *$ age $+0.18, r=0.51, P<.01)$. Figure 2, $A$ shows PIIIP levels in all 4 groups. Serum PIIIP levels were significantly higher in patient groups than in control patients $(0.604 \pm 0.322 \mathrm{U} / \mathrm{mL}$ for $\mathrm{BTS} / \mathrm{PAB}$, $0.176 \pm 0.154 \mathrm{U} / \mathrm{mL}$ for Glenn, $0.143 \pm 0.104 \mathrm{U} / \mathrm{mL}$ for Fontan, and $0.073 \pm 0.129 \mathrm{U} / \mathrm{mL}$ for controls, $P<.01$ for each comparison between the SV group and control group by ANOVA). The difference in the serum PIIIP levels also was observed when the effects of age were taken into account by analysis of covariance $(P<.01$ for group difference). Differences in the serum PIIIP levels between the BTS/PAB and Glenn groups and between the BTS/PAB and Fontan groups were significant $(P<.05$, for both). The difference in serum PIIIP levels between the Glenn and Fontan groups was borderline significant $(P=.08)$. In 45 patients who had serial PIIIP measurements throughout the palliative stages, levels of PIIIP significantly decreased after the surgical stage advanced from BTS/PAB to Fontan (Figure 2, B) (BTS/PAB, $0.671 \pm 0.306 \mathrm{U} / \mathrm{mL}$; Glenn, $0.261 \pm 0.145$ $\mathrm{U} / \mathrm{mL}$; Fontan, $0.173 \pm 0.108 \mathrm{U} / \mathrm{mL} ; P<.01$ between each stage by repeated-measures ANOVA). This demonstrates that tissue fibrogenesis significantly decreased with the surgical stages when ventricular volume load and cyanosis improved. One might suspect that the fibrogenesis response may be different between the single right and single left ventricles, but there were no significant differences between PIIIP values at any surgical stage when compared between the single right and single left ventricles.

TABLE 1. Characteristics of healthy controls and patients with single ventricle circulation

\begin{tabular}{|c|c|c|c|c|}
\hline & Control & BTS/PAB & Glenn & Fontan \\
\hline $\mathrm{n}$ & 149 & 59 & 60 & 53 \\
\hline Male/female & $72 / 77$ & $38 / 21$ & $38 / 22$ & $30 / 23$ \\
\hline Age (y) & $4.3(0.5-10.0)$ & $0.6(0.2-1.9) \dagger$ & $1.9(0.9-3.7) \dagger$ & $4.5(1.7-11.3)$ \\
\hline $\mathrm{BNP}(\mathrm{pg} / \mathrm{mL})$ & $10.1 \pm 13.0$ & $114.1 \pm 148.8 \dagger$ & $63.4 \pm 98.6 \dagger$ & $27.1 \pm 36.4 *$ \\
\hline \multicolumn{5}{|c|}{ Underlying heart diseases (n) } \\
\hline Tricuspid atresia & & 13 & 11 & 9 \\
\hline PA/IVS & & 11 & 10 & 12 \\
\hline \multicolumn{5}{|l|}{ SV } \\
\hline Dominant left & & 6 & 7 & 5 \\
\hline Dominant right & & 13 & 17 & 14 \\
\hline HLHS & & 5 & 5 & 3 \\
\hline Functional SV & & 11 & 10 & 10 \\
\hline \multicolumn{5}{|l|}{ Hemodynamics } \\
\hline Qp/Qs & & $1.6 \pm 0.7$ & - & $0.9 \pm 0.1$ \\
\hline $\mathrm{VP}(\mathrm{mm} \mathrm{Hg})$ & & $84.5 \pm 13.0$ & $84.7 \pm 14.2$ & $100.2 \pm 15.6$ \\
\hline mean PAP (mm Hg) & & $17.3 \pm 7.5$ & $12.3 \pm 3.7$ & $12.9 \pm 2.5$ \\
\hline $\mathrm{SaO}_{2}(\%)$ & & $83.5 \pm 5.1 \ddagger$ & $86.6 \pm 6.1 \dagger$ & $93.4 \pm 5.3$ \\
\hline
\end{tabular}




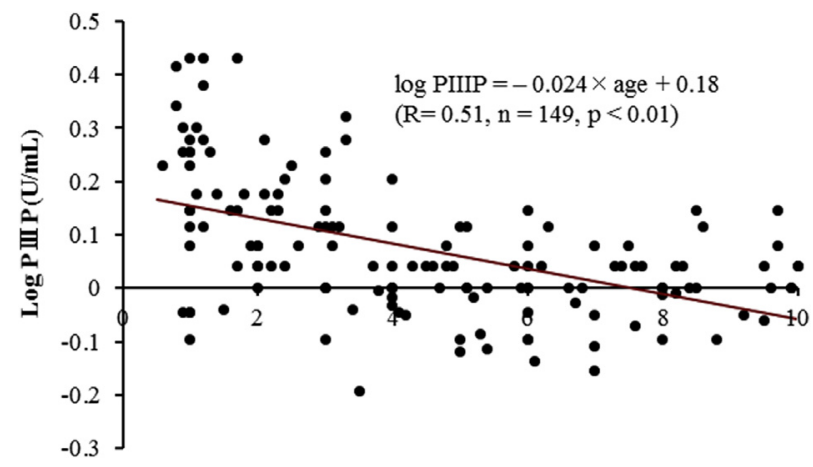

Age (y)

FIGURE 1. Relationship between serum PIIIP levels and age in control patients $(\mathrm{n}=149)$. PIIIP, Amino-terminal procollagen type III.

\section{Determinants of the Serum Amino-Terminal Procollagen Type III Levels}

To further explore whether reduced fibrotic activity is directly associated with improvement of volume load and cyanosis, univariate and multivariate analyses were performed. As shown in Table 2, univariate analysis revealed significant correlations between the serum PIIIP levels with age and Qp/Qs in the BTS/PAB group and with age and $\mathrm{SaO}_{2}$ in the Glenn group. Because the lower levels of $\mathrm{Qp} / \mathrm{Qs}$ inevitably would be associated with lower $\mathrm{SaO}_{2}$ levels in the BTS/PAB group, the effects of cyanosis may have been masked by the counteracting effects of less severe volume load with lower $\mathrm{SaO}_{2}$. In fact, scatter plots for PIIIP versus Qp/Qs (Figure 3, A) appear to show a biphasic change, with increased PIIIP levels at lower levels of $\mathrm{Qp} / \mathrm{Qs}(<1)$. To test this possibility, we performed a nonparametric regression analysis using the random forests method, which is capable of accounting for a nonlinear effect of variables. As shown in Figure 3, B, Qp/Qs had a significant effect on PIIIP levels, after accounting for age. In addition, PIIIP levels showed a U-shaped change according to the $\mathrm{Qp} / \mathrm{Qs}$ values, with the lowest PIIIP levels observed when the Qp/Qs was approximately $1.0(0.87)$, suggesting the potential importance of cyanosis in the elevation of PIIIP in patients with BTS/PAB and low pulmonary flow. In the patients undergoing the Fontan, $\mathrm{SaO}_{2}$ levels did not have a statistically significant correlation with PIIIP levels.

To further test whether the marked reduction in serum PIIIP levels between the BTS/PAB and Fontan groups was biologically relevant, we examined determinants of the altered PIIIP levels ( $\triangle$ PIIIP: PIIIP value after Fontan surgery - PIIIP value after BTS/PAB) by multivariate analysis. Changes in $\mathrm{Qp} / \mathrm{Qs}, \mathrm{SaO}_{2}$, and age $(\Delta \mathrm{Qp} / \mathrm{Qs}$, $\triangle \mathrm{SaO}_{2}$, and $\Delta$ age: value of each variable after Fontan surgery - that after BTS/PAB) and baseline PIIIP values were included as independent variables. We found that $\Delta \mathrm{Qp} / \mathrm{Qs}$ and baseline PIIIP were significant determinants of $\triangle$ PIIIP between BTS and Fontan, with adjustment for $\Delta$ age $(R=0.89, P<.01)$. Furthermore, $\Delta$ PIIIP was significantly correlated with $\triangle \mathrm{BNP}$ (BNP value after Fontan - BNP value after BTS/PAB), a biomarker for cardiac load $(R=0.61, P<.01)$.

\section{Serum Amino-Terminal Procollagen Type III Levels in the Fontan Circulation}

To test whether increased fibrogenesis in the Fontan circulation is associated with ventricular diastolic dysfunction, the correlation between PIIIP and ventricular diastolic function was examined. As shown in Figure $4, A$, there was a significant correlation between serum PIIIP levels and end-diastolic pressure (EDP) in the Fontan group $(\log$ PIIIP $=0.017 \times$ EDP -0.024 ;
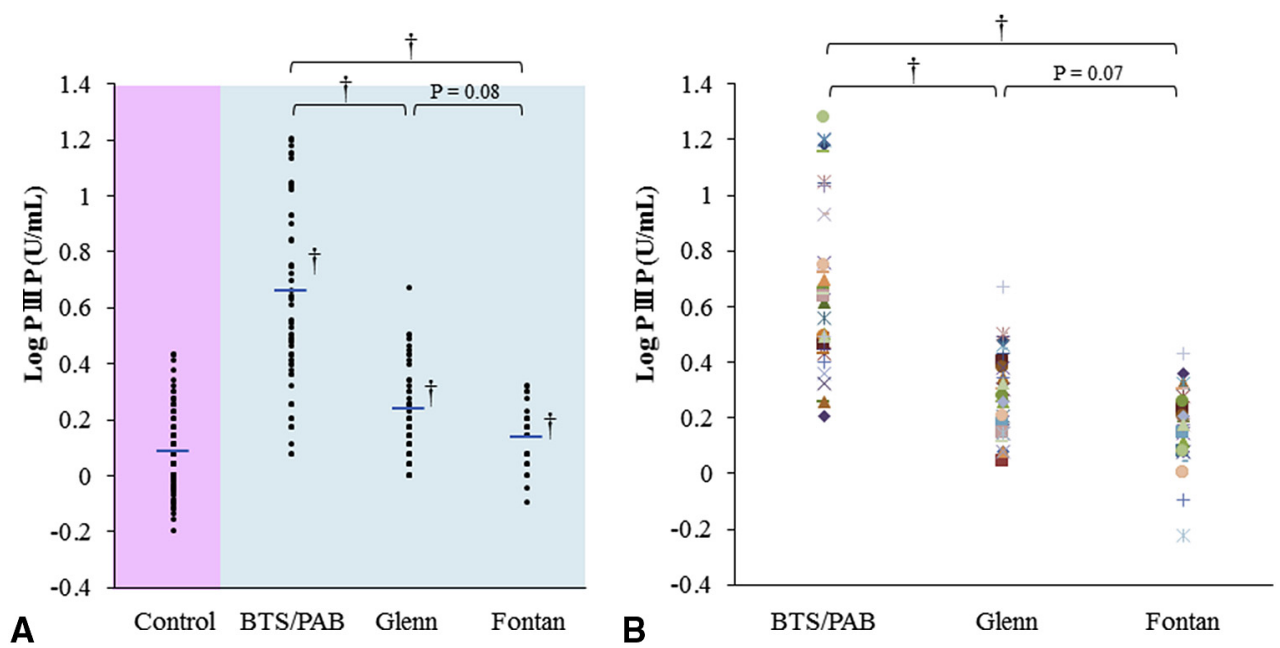

FIGURE 2. A, Serum PIIIP levels for each group. Bars represent the mean values. B, Serial changes in PIIIP levels in the BTS/PAB, Glenn, and Fontan groups. $\dagger P<.05$ versus controls (right shoulder) and between the 2 groups stratified according to age (above the bars). PIIIP, Amino-terminal procollagen type III; $B T S / P A B$, blalock-Taussig shunt/pulmonary artery banding. 
TABLE 2. Univariate and multivariate regression analyses for determinants of serum amino-terminal procollagen type III levels

\begin{tabular}{|c|c|c|c|c|c|c|c|c|c|c|c|c|}
\hline & \multicolumn{4}{|c|}{ BTS/PAB } & \multicolumn{4}{|c|}{ Glenn } & \multicolumn{4}{|c|}{ Fontan } \\
\hline & \multicolumn{2}{|c|}{ Univariate } & \multicolumn{2}{|c|}{ Multivariate } & \multicolumn{2}{|c|}{ Univariate } & \multicolumn{2}{|c|}{ Multivariate } & \multicolumn{2}{|c|}{ Univariate } & \multicolumn{2}{|c|}{ Multivariate } \\
\hline & $r$ & $P$ value & SE & $P$ value & $r$ & $P$ value & SE & $P$ value & $r$ & $P$ value & SE & $P$ value \\
\hline Age & -0.51 & $<.001 *$ & -0.401 & $.002^{*}$ & -0.529 & $<.001 *$ & -0.371 & $.009^{*}$ & -0.204 & .074 & -0.24 & .544 \\
\hline Qp/Qs & 0.508 & $<.001 *$ & 0.399 & $.002 *$ & - & - & - & - & -0.121 & .196 & -0.548 & .276 \\
\hline $\mathrm{VP}(\mathrm{mm} \mathrm{Hg})$ & 0.158 & .141 & 0.158 & .086 & -0.196 & .104 & -0.037 & .774 & -0.127 & .184 & -0.477 & .296 \\
\hline mean PAP $(\mathrm{mm} \mathrm{Hg})$ & 0.173 & .120 & 0.173 & .867 & 0.081 & .302 & 0.185 & .153 & -0.096 & .249 & 0.202 & .534 \\
\hline $\mathrm{SaO}_{2}(\%)$ & 0.105 & .240 & 0.105 & .921 & -0.485 & $<.001 *$ & -0.385 & $.007 *$ & -0.136 & .169 & 0.766 & .129 \\
\hline
\end{tabular}

$B T S / P A B$, Blalock-Taussig shunt/pulmonary artery banding; $S E$, standard error; $Q p / Q s$, pulmonary to systemic blood flow ratio; $V P$, ventricular pressure; $P A P$, pulmonary artery pressure; $\mathrm{SaO}_{2}$, arterial oxygen saturation. ${ }^{*} P<.01$.

$R=0.482, P=.004)$. EDP is an indicator of ventricular dysfunction. A significant relationship between serum PIIIP levels and ventricular stiffness also was observed $(\log$ PIIIP $=0.253 \times \beta+0.030 ; R=0.494$, $P=.009$ ) (Figure $4, B$ ). These relationships also held true after controlling for the age of the patients $(P<.05)$. Patients undergoing the Fontan randomly selected for this particular analysis had demographic and hemodynamic characteristics that were similar to those of the remaining Fontan cases (Table 3); thus, this randomly selected group of patients was representative of the whole Fontan group.

To further elucidate the pathologic significance and prognostic value of PIIIP, we examined whether preFontan PIIIP levels prognosticated post-Fontan diastolic dysfunction. As shown in Figure 5, the pre-Fontan PIIIP values were significantly correlated with levels of post-Fontan EDP $(\log$ PIIIP $=0.013 \times \mathrm{EDP}+0.155$; $R=0.430, P=.040$ ). This correlation persisted even when cardiopulmonary bypass time for the Fontan surgery (133 \pm 34 minutes), a potentially important factor affecting postoperative ventricular function, was taken into account by multivariate analysis $(P=.036$ for PIIIP and $P=.693$ for bypass time).

\section{Renin-Angiotensin-Aldosterone System Activation and Amino-Terminal Procollagen Type III in the Fontan Circulation}

The relationship between serum PIIIP levels and RAS activation in the Fontan group is shown in Figure 4, C-E. All RAS hormones positively correlated with levels of serum PIIIP (renin: $R=0.429, P=.008$; angiotensin II: $R=0.489, P=.002$; aldosterone: $R=0.473, P=.003$ ).

\section{DISCUSSION}

The present study provides the first evidence of increased serum levels of PIIIP, a marker of tissue fibrosis, in children with SV circulation before and even after Fontan surgery. The PIIIP levels decreased with advancing surgical stages as ventricular volume load and cyanosis were alleviated. Indeed, both severity of volume load and cyanosis were important determinants of the increased PIIIP levels in
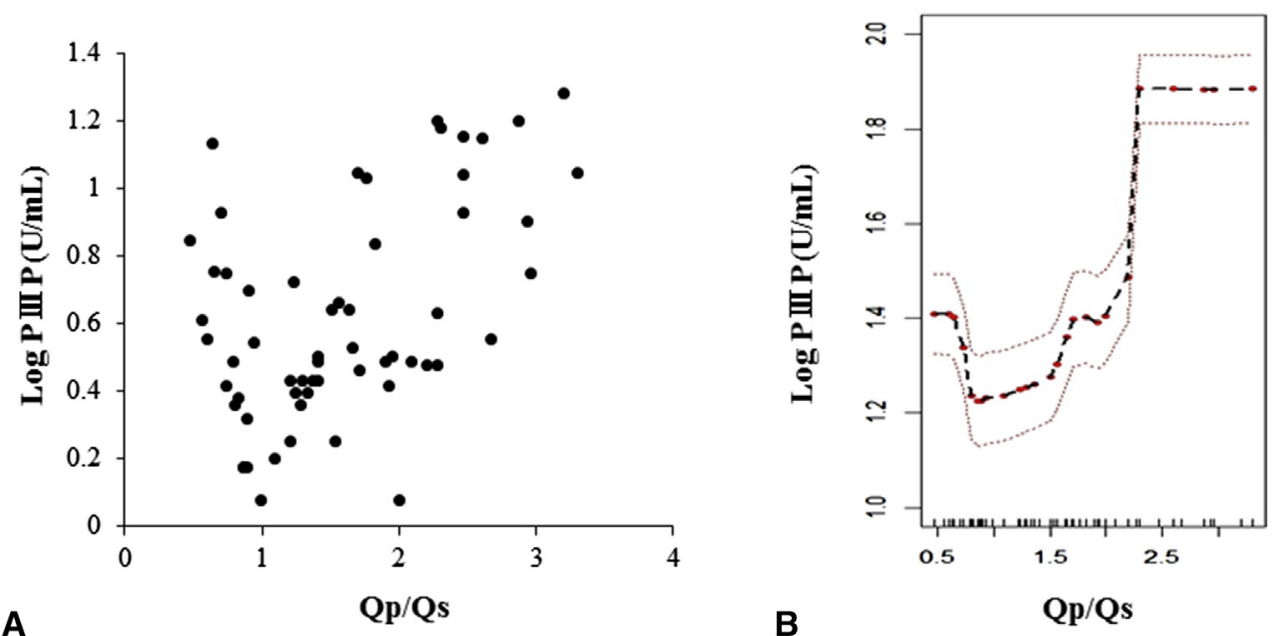

FIGURE 3. A, The relationship between serum PIIIP levels and Qp/Qs in patients in the BTS or PAB group. B, Expected mean PIIIP levels (black dotted line) and 95\% confidence interval of mean PIIIP levels (red dotted line) as a function of Qp/Qs based on random forests regression analysis. $P I I I P$, Amino-terminal procollagen type III; $Q p / Q s$, pulmonary-to-systemic blood flow. 


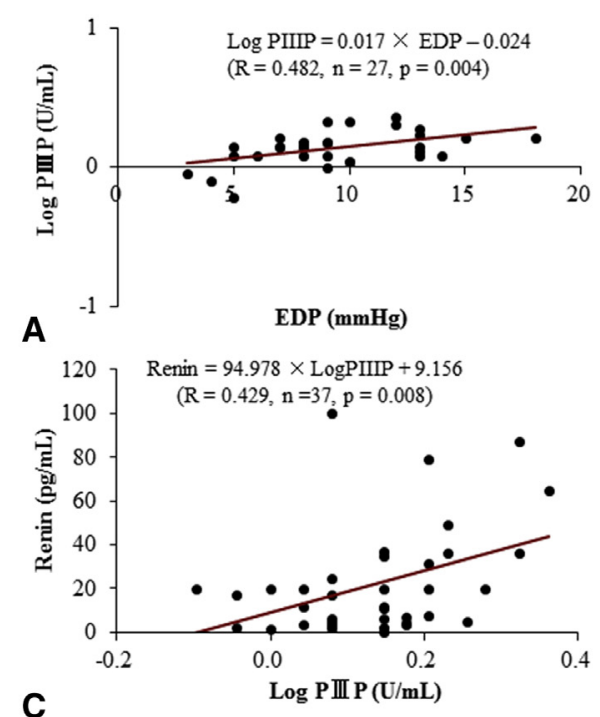

c

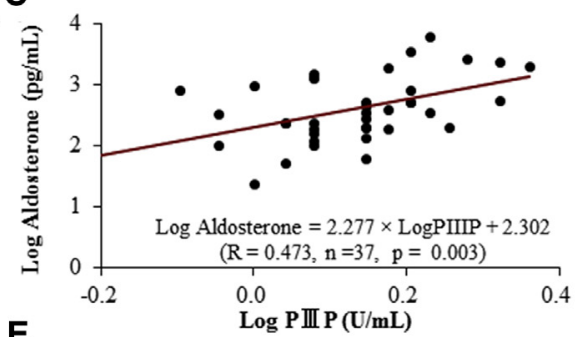

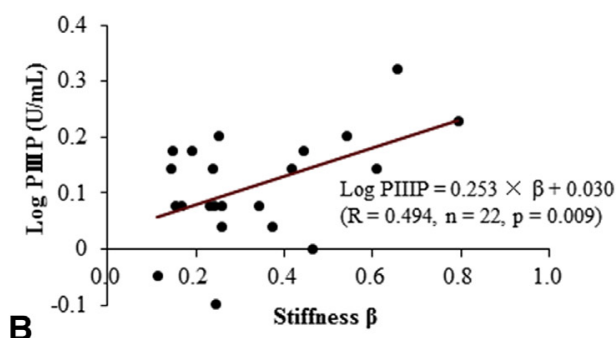

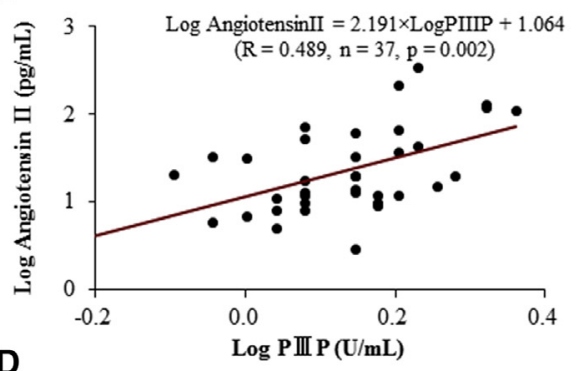

D

FIGURE 4. Relationship between PIIIP levels and EDP $(n=32)(A)$ and ventricular stiffness $(n=22)(B)$ in the Fontan group. Relationship between PIIIP levels and RAS in the Fontan group (C-E). PIIIP, Amino-terminal procollagen type III; EDP, end-diastolic pressure.

patients after BTS or PAB, and the severity of cyanosis was the determining factor of the increased PIIIP in patients with a Glenn circulation when ventricular volume load was largely eliminated. In addition, persistently increased PIIIP even after Fontan surgery was associated with ventricular diastolic stiffening, suggesting the potential importance of ventricular fibrosis in the development of ventricular diastolic dysfunction in patients after Fontan surgery. Data also suggested the potential involvement of RAS activation in the increased fibrogenesis after Fontan surgery. These results not only suggest that serum PIIIP may provide important diagnostic information on myocardial fibrosis but also raise the possibility that ventricular fibrogenesis may be a potential therapeutic target in patients with SV circulation.

\section{Amino-Terminal Procollagen Type III in Single Ventricle Circulation}

It has been demonstrated that the serum levels of PIIIP correlate significantly with myocardial collagen type III contents. ${ }^{15,16}$ Indeed, serum PIIIP levels are significantly elevated in pathologic conditions associated with enhanced myocardial collagen deposition (eg, cardiomyopathy, ${ }^{15,17}$ myocardial infarction, ${ }^{18}$ and hypertension $^{19}$ ) and are decreased in response to therapies that potentially inhibit cardiac fibrosis..$^{17,20,21}$ In addition, several experimental studies have shown that abnormal hemodynamic loading, ${ }^{22}$ hypoxemia, and neurohumoral activation $^{23-25}$ are known to stimulate collagen synthesis, thereby promoting myocardial fibrosis. ${ }^{6,26-30}$ Taken together with the present results, the increased serum PIIIP levels and their close association with hemodynamic load and hypoxia observed in this study strongly suggest increased ventricular fibrosis in patients with SV circulation, and thus serum PIIIP may offer a useful tool for the diagnosis of ventricular fibrotic activity in this population. The present results in patients with SV circulation were consistent with our previous study in simple forms of congenital heart disease, which demonstrated increased serum PIIIP levels in proportion to the magnitude of hemodynamic load or hypoxemia. ${ }^{9}$ Therefore, an increased hemodynamic burden and cyanosis appear to be a common pathway to promote ventricular fibrosis regardless of the type or anatomy of congenital heart disease.

In the BTS/PAB group, both ventricular volume load and cyanosis appeared to be important determinants of fibrotic tissue activity. Of note, PIIIP levels showed a U-shaped change according to the $\mathrm{Qp} / \mathrm{Qs}$ values, with minimum PIIIP levels observed when Qp/Qs was 0.87 (Figure 3, $A$ and $B$ ). 
TABLE 3. Characteristics of Fontan cases with and without stiffness assessment

\begin{tabular}{|c|c|c|c|}
\hline & $\begin{array}{c}\text { P/V loop } \\
\text { examination }\end{array}$ & $\begin{array}{c}\text { Without } \mathrm{P} / \mathrm{V} \\
\text { loop examination }\end{array}$ & $\begin{array}{c}P \\
\text { value }\end{array}$ \\
\hline $\mathrm{n}$ & 22 & 31 & \\
\hline $\mathrm{M} / \mathrm{F}$ & $12 / 10$ & $18 / 13$ & \\
\hline Age (y) & $3.3(1.7-10.0)$ & $3.8(2.1-11.3)$ & \\
\hline $\mathrm{BNP}(\mathrm{pg} / \mathrm{mL})$ & $20.5 \pm 4.0$ & $27.7 \pm 4.0$ & .301 \\
\hline $\operatorname{Renin}(\mathrm{pg} / \mathrm{mL})$ & $14.6 \pm 1.4$ & $25.6 \pm 2.2$ & .063 \\
\hline Log angiotensin II $(\mathrm{pg} / \mathrm{mL})$ & $1.2 \pm 0.8$ & $1.3 \pm 0.5$ & .448 \\
\hline Log aldosterone (pg/mL) & $2.4 \pm 1.4$ & $2.6 \pm 0.6$ & .131 \\
\hline \multicolumn{4}{|l|}{ Underlying heart diseases (n) } \\
\hline Tricuspid atresia & 5 & 4 & \\
\hline PA/IVS & 2 & 10 & \\
\hline \multicolumn{4}{|l|}{ SV } \\
\hline Dominant left & 2 & 3 & \\
\hline Dominant right & 8 & 6 & \\
\hline HLHS & 0 & 3 & \\
\hline Functional SV & 5 & 5 & \\
\hline \multicolumn{4}{|l|}{ Hemodynamics } \\
\hline Qp/Qs & $0.76 \pm 0.11$ & $0.82 \pm 0.17$ & .623 \\
\hline $\mathrm{VP}(\mathrm{mm} \mathrm{Hg})$ & $85.7 \pm 10.3$ & $92.5 \pm 10.6$ & .973 \\
\hline mean PAP $(\mathrm{mm} \mathrm{Hg})$ & $12.3 \pm 8.0$ & $12.4 \pm 3.3$ & 697 \\
\hline $\mathrm{SaO}_{2}(\%)$ & $85.0 \pm 6.3$ & $87.8 \pm 5.6$ & .779 \\
\hline
\end{tabular}

Data are mean \pm standard deviation. $P / V$, Pressure/volume; $B N P$, brain natriuretic peptide; $P A / I V S$, pulmonary artery/intact ventricular septum; $S V$, single ventricle; $H L H S$, hypoplastic left heart syndrome; $Q p / Q s$, pulmonary-to-systemic blood flow; $V P$, ventricular pressure; $P A P$, pulmonary artery pressure; $\mathrm{SaO}_{2}$, arterial oxygen saturation

Thus, Qp/Qs around this value appears to be an optimal shunt ratio when creating $\mathrm{BTS}$ or $\mathrm{PAB}$ in view of minimizing ventricular fibrosis due to coexisting ventricular volume load and cyanosis.

The optimal timing for Fontan completion has become earlier than originally suggested. This is based partly on the assumption that earlier volume unloading is beneficial to preserve ventricular function and thereby reduce late

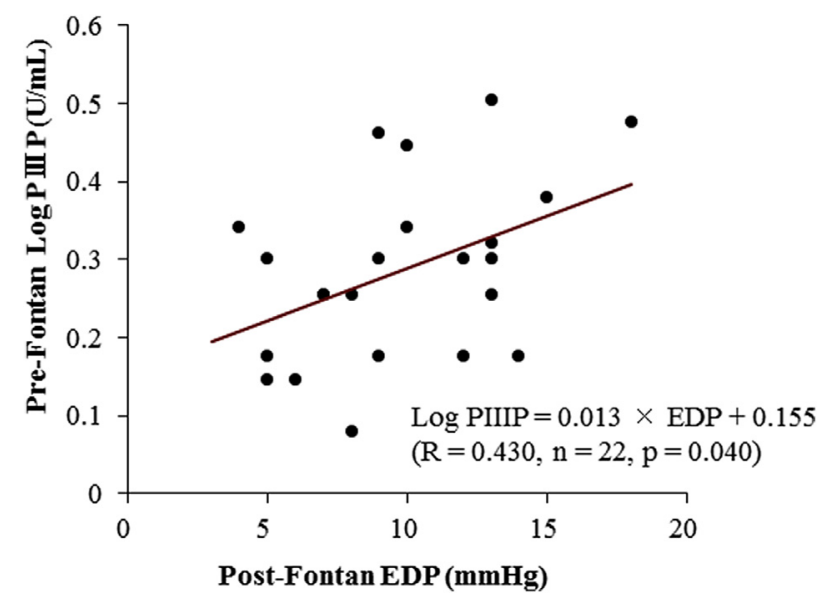

FIGURE 5. Relationship between pre-Fontan PIIIP levels and postFontan ventricular EDP. PIIIP, Amino-terminal procollagen type III; $E D P$, end-diastolic pressure. complications. ${ }^{31,32}$ The present results indicating a progressive decline in PIIIP levels with advancing palliative stages provide a supportive rationale for the prevailing strategy of earlier Fontan completion from the viewpoint of ventricular fibrosis. Conversely, earlier Fontan completion inevitably means that patients must experience a longer duration of elevated venous pressure. Because high venous pressure also is a main cause of late complications after the Fontan operation, ${ }^{33}$ the optimal timing for Fontan completion should be determined with careful consideration of these conflicting effects. Serum PIIIP levels at least may provide useful information about the ventricular structure to help determine the timing of the Fontan surgery.

The results of the present study indicating increased tissue fibrogenesis are consistent with several previous studies demonstrating myocardial fibrosis in patients with SV circulation. Histopathologic studies of tricuspid atresia after various types of palliative surgery, ${ }^{34}$ including BT shunt, Glenn procedure, and Fontan surgery, reported more fibrotic left ventricle than age-matched normal hearts. Likewise, Padalino and colleagues ${ }^{7}$ reported that specimens obtained from patients after the Norwood procedure for hypoplastic left heart syndrome ${ }^{7}$ exhibited increased ventricular fibrosis compared with age- and weight-matched normal hearts. A study using magnetic resonance imaging with late gadolinium enhancement also demonstrated myocardial fibrosis in approximately one third of patients undergoing the Fontan. ${ }^{35}$ No study has been performed in the population with congenital heart disease to directly compare serum PIIIP levels with cardiac magnetic resonance imaging or endomyocardial biopsy. Such studies need to be conducted in the future to further clarify the pathophysiologic meaning of serum PIIIP levels.

\section{Amino-Terminal Procollagen Type III and Fontan Circulation}

Our data also demonstrated that PIIIP levels persistently increased even after the Fontan surgery when volume overload and cyanosis largely had been eliminated. It is well known that ventricular fibrosis is an important pathophysiology underlying ventricular diastolic dysfunction. ${ }^{25,36,37}$ Indeed, several clinical studies have described a close correlation between serum levels of PIIIP and diastolic dysfunction in adults. ${ }^{38}$ Consistent with these experimental and clinical data, we found a significant correlation between increased PIIIP levels and ventricular diastolic stiffening with EDP elevation in patients with Fontan circulation (Figure 4, $A$ and $B$ ). Because ventricular diastolic function may deteriorate with time after Fontan completion, ${ }^{39}$ preventing ventricular fibrosis may offer a way to improve the prognosis of patients after Fontan surgery. In this sense, the relationship between PIIIP and RAS activation (Figure 4, C-E) may suggest the potential efficacy of 
RAS inhibition to prevent ventricular fibrosis after the Fontan surgery. This notion is supported by numerous basic and clinical studies demonstrating the important role of the RAS in the development of myocardial fibrosis and the efficacy of RAS inhibition/blockade for the suppression of myocardial collagen synthesis. ${ }^{19-21}$ Effects of pharmacologic blockade of RAS on serum PIIIP levels, ventricular fibrosis, diastolic function, and prognosis in patients after Fontan surgery warrant further investigation.

\section{Study Limitations}

Ventricular pressure-area analysis to assess ventricular stiffness was performed in only a limited number of patients, although such patients appeared to represent the characteristics of the entire Fontan group (Table 2). Another limitation is the heterogeneous nature of each of the staged subcohorts. Although a significant association between PIIIP values and changes in cardiac load (Qp/Qs), as well as BNP, would support the biological relevance of the observed PIIIP levels, lack of cardiac tissue pathology data directly linking serum PIIIP levels is an important limitation of this study. In this sense, examining PIIIP levels in patients before BTS/PAB would be helpful to further test the biological relevance of serum PIIIP levels. Although we have only limited data for such patients, the average PIIIP value in the 3 patients who had PIIIP measurements before $\mathrm{BTS} / \mathrm{PAB}$ was $0.627 \pm 0.358 \mathrm{U} / \mathrm{mL}$, which was slightly higher than in the BTS/PAB group $(0.604 \pm 0.322$ $\mathrm{U} / \mathrm{mL}$ ). Two of these patients had serial data; PIIIP decreased from 0.74 to $0.49 \mathrm{U} / \mathrm{mL}$ in 1 patient who underwent $\mathrm{PAB}$ and from 0.20 to $0.17 \mathrm{U} / \mathrm{mL}$ in the other patient who underwent BTS. Further accumulation of data is needed. Finally, despite statistically significant differences, there was considerable overlap between PIIIP values among the groups, and the range of PIIIP levels that correlated with diastolic dysfunction was relatively small. Therefore, it is clear that further studies are needed to establish the clinical usefulness of PIIIP as a diagnostic and prognostic marker for quantitatively determining ventricular fibrotic activity. Development of highly sensitive measurement of PIIIP may be helpful.

\section{CONCLUSIONS}

Increased ventricular volume load and cyanosis before the Fontan procedure are likely to be associated with increased ventricular fibrotic activity. The Fontan procedure ameliorates this process, but there is still ongoing fibrogenesis activation that is related to increased ventricular diastolic stiffening and elevated EDP in some patients. The long-term outcomes of increased fibrogenesis and the effects of therapies that potentially suppress this process warrant future study.

\section{Conflict of Interest Statement}

Authors have nothing to disclose with regard to commercial support.

\section{References}

1. Khairy P, Fernandes SM, Mayer JE Jr, Triedman JK, Walsh EP, Lock JE, et al Long-term survival, modes of death, and predictors of mortality in patients with Fontan surgery. Circulation. 2008;117:85-92.

2. Senzaki H, Masutani S, Ishido H, Taketazu M, Kobayashi T, Sasaki N, et al. Cardiac rest and reserve function in patients with Fontan circulation. J Am Coll Cardiol. 2006;47:2528-35.

3. Poh CL, Xu M, Galati JC, Iyengar AJ, Cheung M, Brizard CP, et al. Surgical palliation in patients with a single ventricle and dextrocardia. J Thorac Cardiovasc Surg. 2014;148:1475-80.

4. McLenachan JM, Dargie HJ. Ventricular arrhythmias in hypertensive left ventricular hypertrophy. Relationship to coronary artery disease, left ventricular dysfunction, and myocardial fibrosis. Am J Hypertens. 1990;3:735-40.

5. Swynghedauw B. Molecular mechanisms of myocardial remodeling. Physiol Rev. 1999;79:215-62.

6. Chapman D, Weber KT, Eghbali M. Regulation of fibrillar collagen types I and III and basement membrane type IV collagen gene expression in pressure overloaded rat myocardium. Circ Res. 1990;67:787-94.

7. Padalino MA, Castellani C, Toffoli S, Della Barbera M, Milanesi O, Thiene G, et al. Pathological changes and myocardial remodelling related to the mode of shunting following surgical palliation for hypoplastic left heart syndrome. Cardiol Young. 2008;18:415-22.

8. Poulsen SH, Andersen NH, Heickendorff L, Mogensen CE. Relation between plasma amino-terminal propeptide of procollagen type III and left ventricular longitudinal strain in essential hypertension. Heart. 2005;91:624-9.

9. Sugimoto M, Masutani S, Seki M, Kajino H, Fujieda K, Senzaki H. High serum levels of procollagen type III N-terminal amino peptide in patients with congenital heart disease. Heart. 2009;95:2023-8.

10. Yarbrough WM, Mukherjee R, Ikonomidis JS, Zile MR, Spinale FG. Myocardial remodeling with aortic stenosis and after aortic valve replacement: mechanisms and future prognostic implications. J Thorac Cardiovasc Surg. 2012; 143:656-64.

11. Trivedi P, Cheeseman P, Portmann B, Hegarty J, Mowat AP. Variation in serum type III procollagen peptide with age in healthy subjects and its comparative value in the assessment of disease activity in children and adults with chronic active hepatitis. Eur J Clin Invest. 1985;15:69-74.

12. Risteli J, Niemi S, Trivedi P, Maentausta O, Mowat AP, Risteli L. Rapid equilibrium radioimmunoassay for the amino-terminal propeptide of human type III procollagen. Clin Chem. 1988;34:715-8.

13. Brilla CG, Maisch B, Zhou G, Weber KT. Hormonal regulation of cardiac fibroblast function. Eur Heart J. 1995;16(Suppl C):45-50.

14. Breiman L. Random forests. Mach Learn. 2001;45:5-32.

15. Klappacher G, Franzen P, Haab D, Mehrabi M, Binder M, Plesch K, et al. Measuring extracellular matrix turnover in the serum of patients with idiopathic or ischemic dilated cardiomyopathy and impact on diagnosis and prognosis. Am J Cardiol. 1995;75:913-8.

16. Diez J, Laviades C. Monitoring fibrillar collagen turnover in hypertensive heart disease. Cardiovasc Res. 1997;35:202-5.

17. Tsutamoto T, Wada A, Maeda K, Mabuchi N, Hayashi M, Tsutsui T, et al. Spironolactone inhibits the transcardiac extraction of aldosterone in patients with congestive heart failure. J Am Coll Cardiol. 2000;36:838-44.

18. Jensen LT, Horslev-Petersen K, Toft P, Bentsen KD, Grande P, Simonsen EE, et al. Serum aminoterminal type III procollagen peptide reflects repair after acute myocardial infarction. Circulation. 1990;81:52-7.

19. Diez J, Laviades C, Mayor G, Gil MJ, Monreal I. Increased serum concentrations of procollagen peptides in essential hypertension : relation to cardiac alterations. Circulation. 1995;91:1450-6.

20. Laviades C, Mayor G, Diez J. Treatment with lisinopril normalizes serum concentrations of procollagen type III amino-terminal peptide in patients with essential hypertension. Am J Hypertens. 1994;7:52-8.

21. Hayashi M, Tsutamoto T, Wada A, Tsutsui T, Ishii C, Ohno K, et al. Immediate administration of mineralocorticoid receptor antagonist spironolactone prevents post-infarct left ventricular remodeling associated with suppression of a marker of myocardial collagen synthesis in patients with first anterior acute myocardial infarction. Circulation. 2003;107:2559-65. 
22. Senzaki H, Chen CH, Masutani S, Taketazu M, Kobayashi J, Kobayashi T, et al. Assessment of cardiovascular dynamics by pressure-area relations in pediatric patients with congenital heart disease. J Thorac Cardiovasc Surg. 2001;122: $535-47$.

23. Ratnasamy C, Kinnamon DD, Lipshultz SE, Rusconi P. Associations between neurohormonal and inflammatory activation and heart failure in children. Am Heart J. 2008; 155:527-33.

24. Senzaki H, Gluzband YA, Pak PH, Crow MT, Janicki JS, Kass DA. Synergistic exacerbation of diastolic stiffness from short-term tachycardia-induced cardiodepression and angiotensin II. Circ Res. 1998;82:503-12.

25. Senzaki H, Paolocci N, Gluzband YA, Lindsey ML, Janicki JS, Crow MT, et al. beta-blockade prevents sustained metalloproteinase activation and diastolic stiffening induced by angiotensin II combined with evolving cardiac dysfunction. Circ Res. 2000;86:807-15.

26. Holubarsch C. Contracture type and fibrosis type of decreased myocardial distensibility. Different changes in elasticity of myocardium in hypoxia and hypertrophy. Basic Res Cardiol. 1980;75:244-52.

27. Falanga V, Zhou L, Yufit T. Low oxygen tension stimulates collagen synthesis and COL1A1 transcription through the action of TGF-beta1. J Cell Physiol. 2002;191:42-50.

28. Doering CW, Jalil JE, Janicki JS, Pick R, Aghili S, Abrahams C, et al. Collagen network remodelling and diastolic stiffness of the rat left ventricle with pressure overload hypertrophy. Cardiovasc Res. 1988;22:686-95.

29. Michel JB, Salzmann JL, Ossondo Nlom M, Bruneval P, Barres D, Camilleri JP. Morphometric analysis of collagen network and plasma perfused capillary bed in the myocardium of rats during evolution of cardiac hypertrophy. Basic Res Cardiol. 1986;81:142-54.

30. Kurishima C, Saiki H, Masutani S, Senzaki H. Tailored therapy for aggressive dilatation of systemic veins and arteries may result in improved long-term Fontan circulation. J Thorac Cardiovasc Surg. 2015;150:1367-70.

31. Francois K, Bove T, Panzer J, De Groote K, Vandekerckhove K, De Wilde H, et al. Univentricular heart and Fontan staging: analysis of factors impacting on body growth. Eur J Cardiothorac Surg. 2012;41:e139-45.
32. Dabal RJ, Kirklin JK, Kukreja M, Brown RN, Cleveland DC, Eddins MC, et al. The modern Fontan operation shows no increase in mortality out to 20 years: a new paradigm. J Thorac Cardiovasc Surg. 2014;148:2517-23.e1.

33. Ohuchi H, Yasuda K, Miyazaki A, Kitano M, Sakaguchi H, Yazaki S, et al. Haemodynamic characteristics before and after the onset of protein losing enteropathy in patients after the Fontan operation. Eur J Cardiothorac Surg. 2013;43:e49-57.

34. Ho SY, Jackson M, Kilpatrick L, Smith A, Gerlis LM. Fibrous matrix of ventricular myocardium in tricuspid atresia compared with normal heart. A quantitative analysis. Circulation. 1996;94:1642-6.

35. Rathod RH, Prakash A, Powell AJ, Geva T. Myocardial fibrosis identified by cardiac magnetic resonance late gadolinium enhancement is associated with adverse ventricular mechanics and ventricular tachycardia late after Fontan operation. J Am Coll Cardiol. 2010;55:1721-8.

36. Takeda K, Matsumiya G, Matsue H, Hamada S, Sakaki M, Sakaguchi T, et al. Use of quantitative analysis of remote myocardial fibrosis with delayed-enhancement magnetic resonance imaging to predict outcomes after surgical ventricular restoration for ischemic cardiomyopathy. J Thorac Cardiovasc Surg. 2008;136: 1514-21.

37. Paolocci N, Tavazzi B, Biondi R, Gluzband YA, Amorini AM, Tocchetti CG, et al. Metalloproteinase inhibitor counters high-energy phosphate depletion and AMP deaminase activity enhancing ventricular diastolic compliance in subacute heart failure. J Pharmacol Exp Ther. 2006;317:506-13.

38. Rossi A, Cicoira M, Golia G, Zanolla L, Franceschini L, Marino P, et al. Amino-terminal propeptide of type III procollagen is associated with restrictive mitral filling pattern in patients with dilated cardiomyopathy: a possible link between diastolic dysfunction and prognosis. Heart. 2004;90:650-4.

39. Nakano T, Kado H, Tatewaki H, Hinokiyama K, Oda S, Ushinohama H, et al. Results of extracardiac conduit total cavopulmonary connection in 500 patients $\dagger$. Eur J Cardiothorac Surg. 2015;48:825-32.

Key Words: congenital heart disease, fibrosis, remodeling 